Article

\title{
Synthesis and SAR Studies of Praziquantel Derivatives with Activity against Schistosoma japonicum
}

Wen-Long Wang ${ }^{1,2, \dagger}$, Li-Jun Song ${ }^{3, \dagger}$, Xia Chen ${ }^{1}$, Xu-Ren Yin ${ }^{3}$, Wen-Hua Fan ${ }^{2}$, Gu-Ping Wang ${ }^{1}$, Chuan-Xin $\mathrm{Yu}^{3, *}$ and Bainian Feng ${ }^{1,2, *}$

1 School of Pharmaceutical Science, Jiangnan University, Wuxi 214122, China;

E-Mails: wenlongwang@jiangnan.edu.cn (W.-L.W.); chenxia19891219@126.com (X.C.); p85615822@yeah.net (G.-P.W.)

2 Jiangshu Alpha Biopharmaceuticals, Inc., Wuxi 214122, China;

E-Mail: fanwenhua1987@163.com (W.-H.F.)

3 Key Laboratory on Technology for Parasitic Disease Prevention and Control, Ministry of Health, Jiangsu Institute of Parasitic Diseases, Wuxi 214064, China; E-Mails: songlijun1025@163.com (L.-J.S.); yinxuren@sohu.com (X.-R.Y.)

$\dagger$ These authors contributed equally to this work.

* Authors to whom correspondence should be addressed; E-Mails: chxnyu@163.com (C.-X.Y.); fengbainian@jiangnan.edu.cn (B.F.); Tel./Fax: +86-510-8519-7052 (B.F.).

Received: 25 June 2013; in revised form: 24 July 2013 / Accepted: 25 July 2013 /

Published: 31 July 2013

\begin{abstract}
The synthesis and structure-activity relationship (SAR) studies of praziquantel derivatives with activity against adult Schistosoma japonicum are described. Several of them showed better worm killing activity than praziquantel and could serve as leads for further optimization.
\end{abstract}

Keywords: praziquantel derivatives; antischistosomal activity; Schistosoma japonicum; neglected tropical disease

\section{Introduction}

Schistosomiasis is a neglected tropical disease (NTD) caused by blood-dwelling trematodes belonging to the genus Schistosoma. Schistosoma haematobium, Schistosoma japonicum, and 
Schistosoma mansoni are the main species parasitizing humans [1]. It has been estimated that some 779 million people are at risk for schistosomiasis transmission, with 207 million infected in 76 countries and territories [1,2]. In China, the disease caused by Schistosoma japonicum remains a major public health concern with more than 280 thousand people infected [3]. In the absence of a vaccine, praziquantel (PZQ) has been the only drug recommended by the World Health Organization for the treatment and control of schistosomiasis through mass drug administration (MDA) programs for almost three decades [4]. However, PZQ does not prevent reinfection, and it is inactive against juvenile schistosomes. Furthermore, it has only a limited effect on developed liver and spleen lesions, as well as on the emergent schistosome phenotypes that are resistant to PZQ chemotherapy $[5,6]$. Therefore, it is imperative to develop new antischistosomal agents. A number of recent studies were directed towards exploration of new chemical entities from natural products [7-10] and towards the identification of additional drug targets for schistosomiasis [11-15].

In addition, there are two other major issues with regard to PZQ: (1) the mechanism of action of PZQ is uncertain [16] and (2) as currently administered, PZQ is a racemic mixture [16]. It is a great challenge to prepare (-)-PZQ inexpensively and stereoselectively [17]. The less active (+)-PZQ enantiomer has well been documented as having a bitter and disgusting taste [18]. Recently, many synthetic routes for PZQ and its derivatives have been developed [19,20], providing more opportunities to make novel PZQ derivatives as potential drugs for the treatment of schistosomiasis.

Figure 1. Chemical structure of praziquanel (PZQ) and some reported derivatives.

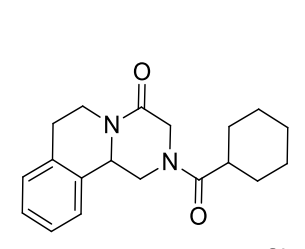

$P Z Q$

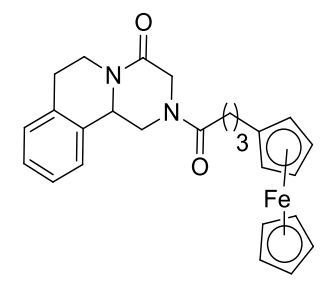

Fc-PZQ derivative from Ref 22

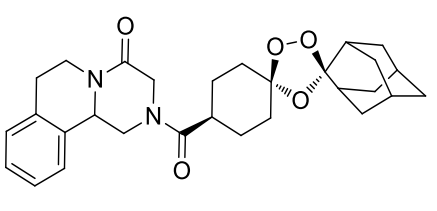

PZQ-Ozonide hybrid from Ref 23

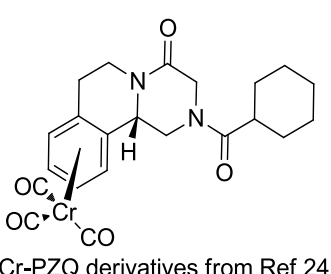

Cr-PZQ derivatives from Ref 24

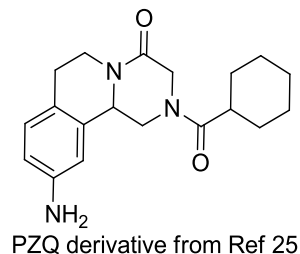

PZQ derivative from Ref 25

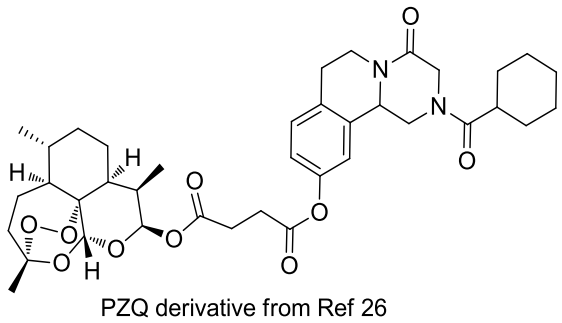

$P Z Q$ derivative from Ref 26

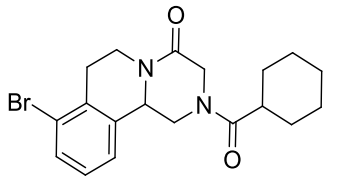

Aromatic ring modified $\mathrm{PZQ}$ derivative from Ref 27

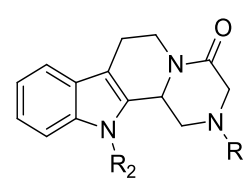

PZQ derivatives from Ref 28

Ever since the discovery of PZQ, there has been interest in the elucidation of the structure-activity relationships (SAR) [21], mostly from data generated using S. mansoni, and to some extent with data for S. japonicum. There are five positions amenable for chemical modification in the PZQ molecular structure [16,22] (Figure 1). Among these, only position $\mathrm{R}_{1}$ has been heavily investigated [21]. Recently, more variants at the $\mathrm{R}_{1}$ position as well as PZQ-ozonide hybrids were synthesized and tested against juvenile and adult stages of $S$. mansoni in vitro and in vivo. Unfortunately, all the variants showed decreased activity compared to the parent compound PZQ [23]. Patra and co-workers reported PZQ 
analogues that replaced the cyclohexyl group at position 2 with different ferrocenyl moieties. Unfortunately, the Fc-PZQ derivatives were considerably less active than PZQ when tested against S. mansoni in vitro [22]. Recently, Gasser and coworkers reported Cr-PZQ derivatives with comparable antischistosomal activities to PZQ when tested against adult S. mansoni [24]. In 2007, the Todd group first reported several derivatives through nitration and amination of the $\mathrm{C} 10$ position of the aromatic ring on PZQ. However, these compounds showed significantly decreased worm killing activity against $S$. mansoni in vitro [25]. In 2012, the Qiao group synthesized eight PZQ/peroxide conjugates, with some compounds showing superior worm-killing activity against $S$. japonicum in in vitro assays compared to PZQ [26]. Recently, the Qiao group reported aromatic ring-modified praziquantel derivatives with activity against both juvenile and adult $S$. japonicum, and a compound with a bromide atom at C8 position showed higher potency against adult $S$. japonicum than PZQ in vitro and comparable antischistosomal activity to PZQ in vivo. However, none of them showed superior worm-killing ability to PZQ [27]. The Rao group developed an efficient synthetic route to provide structurally diverse analogues for SAR studies. Several PZQ analogues with an indol ring were synthesized and tested against adult $S$. mansoni. Unfortunately, no compounds showed better activity than PZQ in vitro [28]. Taken together, all this data indicates that although much work has been done on PZQ, it is still necessary to further expand the range of PZQ analogues in order to obtain chemical probes to elucidate the real biological mechanism of $P Z Q$, and to generate alternative new chemotherapies. Therefore, we became interested in the synthesis of new PZQ analogues to find new potential antischistosomal agents against S. japonicum.

\section{Results and Discussion}

\subsection{Chemistry}

Due to the lack of SAR details from S. japonicum, we initially resynthesized some PZQ analogues with position 2 substitution and evaluated their worm-killing activities against $S$. japonicum (Scheme 1). The racemic PZQ from Aldrich was hydrolyzed by $2 \mathrm{~N} \mathrm{HCl}$ to yield praziquanamine (1) using the method described by the Vennerstrom group [23], followed by coupling with acyl chlorides to yield compounds 2-12. Reductive amination reactions of appropriate aldehydes with praziquanamine (1) were performed with sodium triacetoxyborohydride in dichloroethane at room temperature to afford compounds 13-15 in yields ranging from $27 \%$ to $35 \%$. Praziquanamine (1) was reduced by lithium aluminum hydride to yield compound 16, followed by acylation to yield compounds 17-22. To extend the SAR studies, the aromatic moiety was replaced by a thiophene ring, and compound $\mathbf{2 3}$ was synthesized using the method described by Frehel and his coworkers [29]. Compound 23 was hydrolyzed with $2 \mathrm{~N} \mathrm{HCl}$ to yield amine $\mathbf{2 4}$, followed by acylation with appropriate acyl chlorides to yield compounds 25-33 (Scheme 2). As in previous reports [22], we also observed the major isomer in mixtures with its minor enantiomer with respect to the $11 \mathrm{~b}$ position on the ring system for compounds 1-33. The results were also in agreement with the nature of the commercially available PZQ [19,30] and its thiophene analogue $\mathbf{2 3}$ [20]. 
Scheme 1. Synthesis of PZQ analogues 1-22.

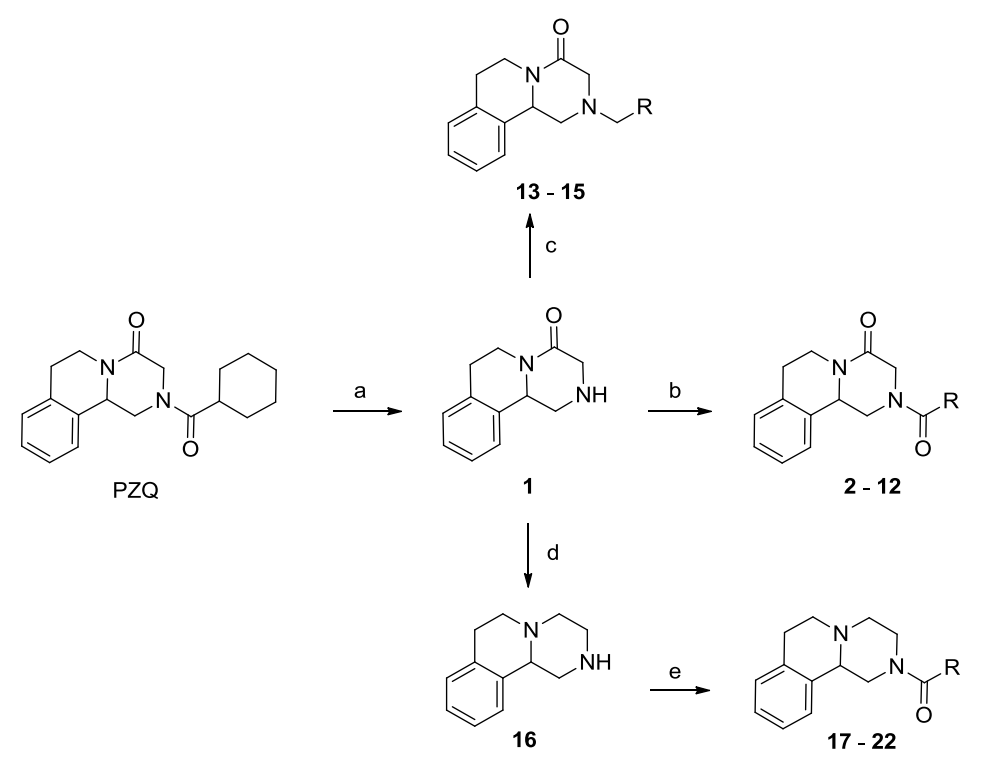

Reagents and conditions: (a) $2 \mathrm{~N} \mathrm{HCl}$, reflux, overnight, $62 \%$; (b) TFAA, $\mathrm{Et}_{3} \mathrm{~N}, \mathrm{DCM}, 62 \%$ or $\mathrm{RCOCl} \mathrm{Et}_{3} \mathrm{~N}$, DCM, $29 \%-50 \%$; (c) $\mathrm{RCHO}, \mathrm{NaBH}_{4}, \mathrm{HOAc}, \mathrm{MeOH}, 0{ }^{\circ} \mathrm{C}$ to $60{ }^{\circ} \mathrm{C}, 27 \%-35 \%$; (d) $\mathrm{LiAlH}_{4}, \mathrm{THF}, 0{ }^{\circ} \mathrm{C}, 35 \%$; (e) $\mathrm{RCOCl}, \mathrm{Et}_{3} \mathrm{~N}, \mathrm{DCM}, 32 \%-69 \%$.

Scheme 2. Synthesis of PZQ analogues 24-33.

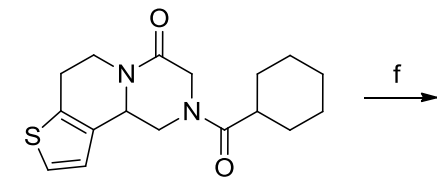

23

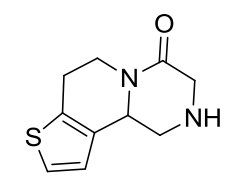

24

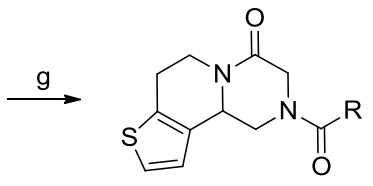

$25-33$

Reagents and conditions: (f) $2 \mathrm{~N} \mathrm{HCl}$, reflux, overnight, $78 \%$; (g) RCOCl, $\mathrm{Et}_{3} \mathrm{~N}, \mathrm{DCM}, 40 \%-76 \%$.

\subsection{Biological Activities}

The biological results are listed in Table 1. Among compounds with an aliphatic cyclic ring (2-4 and PZQ), the worm-killing activity was improved by increasing the ring size. Compounds with an acetyl group (i.e., 5) and with an isobutyryl group (i.e., 6) lacked worm-killing activity. Among compounds with a halogen atom, compound 7 with the chloroacetyl group killed $100 \%$ of the worms at the concentration of $5 \mu \mathrm{M}$, showing better activity than PZQ. However, compound 8 with a 2,2,2-trifluoroacetyl group lost worm killing activity. Replacement of the cyclohexyl ring with a heterocyclic ring, (compound $\mathbf{1 1}$ with a thiophene-2-carbonyl group) led to a similar activity to that of PZQ. However, compound $\mathbf{1 0}$ with an isonicotinoyl group and compound $\mathbf{1 2}$ with a furan-2-carbonyl group did not show any worm-killing activity below the concentration of $100 \mu \mathrm{M}$. Compound $\mathbf{9}$, with a benzoyl group, showed similar activity as PZQ. Replacement of the carbonyl group at position 2 with $\mathrm{CH}_{2}$ gave compounds 13-15, which showed no worm-killing activity. The results indicated that the amide bond at position 2 is essential for activity against $S$. japonicum. The SAR results displayed similarities between compounds 17-22 and compounds containing amide (3, 4, PZQ, 9, 11, and 7), showing decreased activity. The results indicated that the amide bond at position 4 was very important 
to maintain the activity of PZQ derivatives. The SAR results from $S$. japonicum adult worms were similar with those from $S$. mansoni [21]. However, it still gave us valuable information to explore novel PZQ derivatives as antischistosomal agents.

To enlarge the chemical space of potential drug candidates, replacement of the phenyl ring on PZQ with a thiophene ring gave compounds 23-33. Among compounds with an alkyl group (i.e., 23, 25-29), compound $\mathbf{2 3}$ with a cyclohexanecarbonyl group showed the best worm-killing activity, and it showed similar activity to PZQ. However, the worm-killing activity of compounds 24-29 was dramatically decreased. The amine-containing compound $\mathbf{2 4}$ had no activity. Among compounds with an aromatic ring, compound $\mathbf{3 0}$ with a benzoyl group showed slight worm-killing activity, while compound $\mathbf{3 1}$ with a thiophene-2-carbonyl group and compound 32 with a furan-2-carbonyl group had no activity. Interestingly, compound $\mathbf{3 3}$ with a chloroacetyl group showed good worm killing activity at $10 \mu \mathrm{M}$, similar to PZQ derivative 7. Among the compounds reported here, compounds 7, 22 and 33 with a chloroacetyl group showed better antischistosomal activity than PZQ. The evaluation of their cytotoxity is in progress. There have been many compounds with a chloroacetyl group under development [31-33]. For example, TNP470 [O-(chloroacetylcarbamoyl)fumagillol], which was derived from the natural product fumagillin, was in clinical trials for Kaposi's sarcoma [34], as well as renal, brain, breast, cervical and prostate cancers [35].

Table 1. Worm-killing activity on S. japonicum adult worms in vitro by compounds 1-33.

\begin{tabular}{|c|c|c|c|c|c|c|c|c|}
\hline \multirow[t]{2}{*}{ Compound } & \multirow[t]{2}{*}{$\mathbf{R}$} & \multirow[t]{2}{*}{$\mathbf{X}$} & \multirow[t]{2}{*}{$\mathbf{Y}$} & \multirow[t]{2}{*}{$\mathbf{Z}$} & \multicolumn{4}{|c|}{ Killing activity $^{\text {a }}$} \\
\hline & & & & & Conc $(\mu M)^{b}$ & $24 \mathrm{~h}$ & $48 \mathrm{~h}$ & $72 \mathrm{~h}$ \\
\hline Vehicle & & & & & & n.e & n.e & n.e \\
\hline \multirow[t]{4}{*}{ PZQ } & & & & & 10 & $25.0 \% \mathrm{D}$ & $25.0 \% \mathrm{D}$ & $37.5 \% \mathrm{D}$ \\
\hline & & & & & 25 & $25.0 \% \mathrm{D}$ & $25.0 \% \mathrm{D}$ & $62.5 \% \mathrm{D}$ \\
\hline & & & & & 50 & $25.0 \% \mathrm{D}$ & $37.5 \% \mathrm{D}$ & $62.5 \% \mathrm{D}$ \\
\hline & & & & & 100 & $37.5 \% \mathrm{D}$ & $50.0 \% \mathrm{D}$ & $87.5 \% \mathrm{D}$ \\
\hline $\mathbf{1}^{\mathrm{d}, \mathrm{e}}$ & None & $\mathrm{O}$ & $\mathrm{CH}=\mathrm{CH}$ & None & 100 & n.e. & n.e. & n.e. \\
\hline $2^{\mathrm{d}, \mathrm{e}}$ & $\nabla$ & $\mathrm{O}$ & $\mathrm{CH}=\mathrm{CH}$ & $\mathrm{O}$ & 100 & sluggish & $25.0 \%$ & $75.0 \%$ \\
\hline \multirow[t]{4}{*}{$3^{\mathrm{e}}$} & $\square$ & $\mathrm{O}$ & $\mathrm{CH}=\mathrm{CH}$ & $\mathrm{O}$ & 10 & n.e. & n.e. & sluggish \\
\hline & & & & & 25 & n.e. & n.e. & $10.0 \% \mathrm{D}$ \\
\hline & & & & & 50 & n.e. & sluggish & $75.0 \% \mathrm{D}$ \\
\hline & & & & & 100 & sluggish & sluggish & $87.5 \% \mathrm{D}$ \\
\hline \multirow[t]{4}{*}{$4^{\mathrm{e}}$} & & $\mathrm{O}$ & $\mathrm{CH}=\mathrm{CH}$ & $\mathrm{O}$ & 10 & sluggish & sluggish & $37.5 \% \mathrm{D}$ \\
\hline & & & & & 25 & sluggish & sluggish & $50.0 \% \mathrm{D}$ \\
\hline & & & & & 50 & sluggish & sluggish & $100 \% \mathrm{D}$ \\
\hline & & & & & 100 & $12.5 \% \mathrm{D}$ & $12.5 \% \mathrm{D}$ & $75.0 \% \mathrm{D}$ \\
\hline
\end{tabular}


Table 1. Cont.

\begin{tabular}{|c|c|c|c|c|c|c|c|c|}
\hline \multirow[t]{2}{*}{ Compound } & \multirow[t]{2}{*}{$\mathbf{R}$} & \multirow[t]{2}{*}{$\mathbf{X}$} & \multirow[t]{2}{*}{$\mathbf{Y}$} & \multirow[t]{2}{*}{$\mathbf{Z}$} & \multicolumn{4}{|c|}{ Killing activity ${ }^{a}$} \\
\hline & & & & & Conc $(\mu M)^{b}$ & $24 \mathrm{~h}$ & $48 \mathrm{~h}$ & $72 \mathrm{~h}$ \\
\hline $5^{\mathrm{d}, \mathrm{e}}$ & $\mathrm{CH}_{3}$ & $\mathrm{O}$ & $\mathrm{CH}=\mathrm{CH}$ & $\mathrm{O}$ & 100 & n.e. & n.e. & n.e. \\
\hline $6^{\mathrm{d}, \mathrm{e}}$ & & $\mathrm{O}$ & $\mathrm{CH}=\mathrm{CH}$ & $\mathrm{O}$ & 100 & n.e. & n.e. & $20.0 \% \mathrm{D}$ \\
\hline \multirow[t]{8}{*}{$7^{\mathrm{c}, \mathrm{e}}$} & \multirow{8}{*}{$\mathrm{ClCH}_{2}$} & \multirow[t]{8}{*}{$\mathrm{O}$} & \multirow[t]{8}{*}{$\mathrm{CH}=\mathrm{CH}$} & \multirow[t]{8}{*}{$\mathrm{O}$} & 1 & n.e. & n.e. & n.e. \\
\hline & & & & & 3 & n.e. & $25.0 \% \mathrm{D}$ & $25.0 \% \mathrm{D}$ \\
\hline & & & & & 5 & $70.0 \% \mathrm{D}$ & $90.0 \% \mathrm{D}$ & $100 \% \mathrm{D}$ \\
\hline & & & & & 8 & $100 \% \mathrm{D}$ & $100 \% \mathrm{D}$ & $100 \% \mathrm{D}$ \\
\hline & & & & & 10 & $100 \% \mathrm{D}$ & $100 \% \mathrm{D}$ & $100 \% \mathrm{D}$ \\
\hline & & & & & 25 & $100 \% \mathrm{D}$ & $100 \% \mathrm{D}$ & $100 \% \mathrm{D}$ \\
\hline & & & & & 50 & $100 \% \mathrm{D}$ & $100 \% \mathrm{D}$ & $100 \% \mathrm{D}$ \\
\hline & & & & & 100 & $100 \% \mathrm{D}$ & $100 \% \mathrm{D}$ & $100 \% \mathrm{D}$ \\
\hline $8^{\mathrm{d}, \mathrm{e}}$ & & $\mathrm{O}$ & $\mathrm{CH}=\mathrm{CH}$ & $\mathrm{O}$ & 100 & n.e. & n.e. & n.e. \\
\hline \multirow{4}{*}{$9^{\mathrm{e}}$} & & \multirow[t]{4}{*}{$\mathrm{O}$} & \multirow[t]{4}{*}{$\mathrm{CH}=\mathrm{CH}$} & \multirow[t]{4}{*}{$\mathrm{O}$} & 10 & n.e. & n.e. & $55.6 \% \mathrm{D}$ \\
\hline & & & & & 25 & sluggish & $25.0 \% \mathrm{D}$ & $87.5 \% \mathrm{D}$ \\
\hline & & & & & 50 & $18.2 \% \mathrm{D}$ & $36.4 \% \mathrm{D}$ & $45.5 \% \mathrm{D}$ \\
\hline & & & & & 100 & $45.5 \% \mathrm{D}$ & $45.5 \% \mathrm{D}$ & $81.8 \% \mathrm{D}$ \\
\hline $10^{\mathrm{d}, \mathrm{e}}$ & & $\mathrm{O}$ & $\mathrm{CH}=\mathrm{CH}$ & $\mathrm{O}$ & 100 & n.e. & n.e. & sluggish \\
\hline \multirow{4}{*}{$11^{\mathrm{e}}$} & & \multirow[t]{4}{*}{$\mathrm{O}$} & \multirow[t]{4}{*}{$\mathrm{CH}=\mathrm{CH}$} & \multirow[t]{4}{*}{$\mathrm{O}$} & 10 & n.e. & n.e. & $62.5 \% \mathrm{D}$ \\
\hline & & & & & 25 & n.e. & n.e. & $62.5 \% \mathrm{D}$ \\
\hline & & & & & 50 & $25.0 \% \mathrm{D}$ & $37.5 \% \mathrm{D}$ & $87.5 \% \mathrm{D}$ \\
\hline & & & & & 100 & $14.3 \% \mathrm{D}$ & $28.6 \% \mathrm{D}$ & $87.5 \% \mathrm{D}$ \\
\hline $12^{\mathrm{d}, \mathrm{e}}$ & & $\mathrm{O}$ & $\mathrm{CH}=\mathrm{CH}$ & $\mathrm{O}$ & 100 & n.e. & n.e. & n.e. \\
\hline $13^{d}$ & & $\mathrm{O}$ & $\mathrm{CH}=\mathrm{CH}$ & $\mathrm{H}_{2}$ & 100 & n.e. & n.e. & n.e. \\
\hline $14^{\mathrm{d}, \mathrm{e}}$ & & $\mathrm{O}$ & $\mathrm{CH}=\mathrm{CH}$ & $\mathrm{H}_{2}$ & 100 & n.e. & n.e. & n.e. \\
\hline $15^{\mathrm{d}}$ & & $\mathrm{O}$ & $\mathrm{CH}=\mathrm{CH}$ & $\mathrm{H}_{2}$ & 100 & n.e. & n.e. & n.e. \\
\hline $16^{\mathrm{d}, \mathrm{e}}$ & None & $\mathrm{H}_{2}$ & $\mathrm{CH}=\mathrm{CH}$ & None & 100 & n.e. & n.e. & n.e. \\
\hline $17^{\mathrm{d}}$ & & $\mathrm{H}_{2}$ & $\mathrm{CH}=\mathrm{CH}$ & $\mathrm{O}$ & 100 & n.e. & n.e. & n.e. \\
\hline \multirow{4}{*}{18} & & \multirow[t]{4}{*}{$\mathrm{H}_{2}$} & \multirow[t]{4}{*}{$\mathrm{CH}=\mathrm{CH}$} & \multirow[t]{4}{*}{$\mathrm{O}$} & 10 & sluggish & sluggish & sluggish \\
\hline & & & & & 25 & sluggish & $18.2 \% \mathrm{D}$ & $18.2 \% \mathrm{D}$ \\
\hline & & & & & 50 & $10.0 \% \mathrm{D}$ & $20.0 \% \mathrm{D}$ & $50.0 \% \mathrm{D}$ \\
\hline & & & & & 100 & $25.0 \% \mathrm{D}$ & $25.0 \% \mathrm{D}$ & $75.0 \% \mathrm{D}$ \\
\hline \multirow[t]{4}{*}{19} & & $\mathrm{H}_{2}$ & $\mathrm{CH}=\mathrm{CH}$ & $\mathrm{O}$ & 10 & n.e. & n.e. & n.e. \\
\hline & & & & & 25 & n.e. & $14.3 \% \mathrm{D}$ & $57.1 \% \mathrm{D}$ \\
\hline & & & & & 50 & sluggih & $33.3 \% \mathrm{D}$ & $66.7 \% \mathrm{D}$ \\
\hline & & & & & 100 & sluggish & sluggish & $85.7 \% \mathrm{D}$ \\
\hline $\mathbf{2 0}^{\mathrm{d}, \mathrm{e}}$ & & $\mathrm{H}_{2}$ & $\mathrm{CH}=\mathrm{CH}$ & $\mathrm{O}$ & 100 & n.e. & n.e. & n.e. \\
\hline $21^{\mathrm{d}}$ & & $\mathrm{H}_{2}$ & $\mathrm{CH}=\mathrm{CH}$ & $\mathrm{O}$ & 100 & n.e. & n.e. & n.e. \\
\hline 22 & $\mathrm{ClCH}_{2}$ & $\mathrm{H}_{2}$ & $\mathrm{CH}=\mathrm{CH}$ & $\mathrm{O}$ & 10 & n.e. & sluggish & $50.0 \% \mathrm{D}$ \\
\hline & & & & & 25 & $87.5 \% \mathrm{D}$ & $100 \% \mathrm{D}$ & $100 \% \mathrm{D}$ \\
\hline & & & & & 50 & $100 \% \mathrm{D}$ & $100 \% \mathrm{D}$ & $100 \% \mathrm{D}$ \\
\hline & & & & & 100 & $100 \% \mathrm{D}$ & $100 \% \mathrm{D}$ & $100 \% \mathrm{D}$ \\
\hline
\end{tabular}


Table 1. Cont.

\begin{tabular}{|c|c|c|c|c|c|c|c|c|}
\hline \multirow[t]{2}{*}{ Compound } & \multirow[t]{2}{*}{$\mathbf{R}$} & \multirow[t]{2}{*}{$\mathbf{X}$} & \multirow[t]{2}{*}{$\mathbf{Y}$} & \multirow[t]{2}{*}{$\mathbf{Z}$} & \multicolumn{4}{|c|}{ Killing activity ${ }^{a}$} \\
\hline & & & & & $\operatorname{Conc}(\mu \mathrm{M})^{\mathrm{b}}$ & $24 \mathrm{~h}$ & $48 \mathrm{~h}$ & $72 \mathrm{~h}$ \\
\hline \multirow[t]{4}{*}{$23^{\mathrm{e}}$} & & $\mathrm{O}$ & $\mathrm{S}$ & $\mathrm{O}$ & 10 & sluggish & $75.0 \% \mathrm{D}$ & $75.0 \% \mathrm{D}$ \\
\hline & & & & & 25 & sluggish & $87.5 \% \mathrm{D}$ & $87.5 \% \mathrm{D}$ \\
\hline & & & & & 50 & $25.0 \%$ & $75.0 \% \mathrm{D}$ & $87.5 \% \mathrm{D}$ \\
\hline & & & & & 100 & $28.6 \% \mathrm{D}$ & $71.4 \% \mathrm{D}$ & $71.4 \% \mathrm{D}$ \\
\hline $24^{\mathrm{d}}$ & None & $\mathrm{O}$ & $\mathrm{S}$ & None & 100 & n.e. & n.e. & n.e. \\
\hline $25^{d}$ & & $\mathrm{O}$ & $\mathrm{S}$ & $\mathrm{O}$ & 100 & n.e. & $12.5 \% \mathrm{D}$ & $37.5 \% \mathrm{D}$ \\
\hline $26^{d}$ & & $\mathrm{O}$ & $\mathrm{S}$ & $\mathrm{O}$ & 100 & n.e. & sluggish & sluggish \\
\hline $27^{\mathrm{d}}$ & $\triangleright$ & $\mathrm{O}$ & $\mathrm{S}$ & $\mathrm{O}$ & 100 & n.e. & n.e. & sluggish \\
\hline $28^{\mathrm{d}}$ & & $\mathrm{O}$ & $\mathrm{S}$ & $\mathrm{O}$ & 100 & n.e. & n.e. & n.e. \\
\hline $29^{\mathrm{d}}$ & & $\mathrm{O}$ & $\mathrm{S}$ & $\mathrm{O}$ & 100 & sluggish & sluggish & sluggish \\
\hline \multirow[t]{4}{*}{$30^{\mathrm{e}}$} & & $\mathrm{O}$ & $\mathrm{S}$ & $\mathrm{O}$ & 10 & n.e. & n.e. & n.e. \\
\hline & & & & & 25 & n.e. & sluggish & sluggish \\
\hline & & & & & 50 & n.e. & $14.3 \% \mathrm{D}$ & $14.3 \% \mathrm{D}$ \\
\hline & & & & & 100 & sluggish & $37.5 \% \mathrm{D}$ & $75.0 \% \mathrm{D}$ \\
\hline $31^{\mathrm{d}, \mathrm{e}}$ & T & $\mathrm{O}$ & $\mathrm{S}$ & $\mathrm{O}$ & 100 & n.e. & n.e. & n.e. \\
\hline $\mathbf{3 2}^{\mathrm{d}, \mathrm{e}}$ & ¿17 & $\mathrm{O}$ & $\mathrm{S}$ & $\mathrm{O}$ & 100 & n.e. & n.e. & n.e. \\
\hline \multirow[t]{8}{*}{$3^{c}{ }^{c}$} & $\mathrm{ClCH}_{2}$ & $\mathrm{O}$ & $\mathrm{S}$ & $\mathrm{O}$ & 1 & n.e. & n.e. & n.e. \\
\hline & & & & & 3 & n.e. & n.e. & n.e. \\
\hline & & & & & 5 & sluggish & $25 \% \mathrm{D}$ & $37.5 \% \mathrm{D}$ \\
\hline & & & & & 8 & $75 \% \mathrm{D}$ & $87.5 \% \mathrm{D}$ & $87.5 \% \mathrm{D}$ \\
\hline & & & & & 10 & $100 \% \mathrm{D}$ & $100 \% \mathrm{D}$ & $100 \% \mathrm{D}$ \\
\hline & & & & & 25 & $100 \% \mathrm{D}$ & $100 \% \mathrm{D}$ & $100 \% \mathrm{D}$ \\
\hline & & & & & 50 & $100 \% \mathrm{D}$ & $100 \% \mathrm{D}$ & $100 \% \mathrm{D}$ \\
\hline & & & & & 100 & $100 \% \mathrm{D}$ & $100 \% \mathrm{D}$ & $100 \% \mathrm{D}$ \\
\hline
\end{tabular}

${ }^{a}$ Data collected by visual examination of worm movement and shape (the values were the averages of two tests); n.e. (no effect): all worms are scored as active in culture with typical appearance; sluggish: worm movement is significantly reduced; \% D = number of dead worms/total number of worms observed, and dead worms judged by lack of movement within 2 minutes in addition to morphological and tegumental alterations;

${ }^{\mathrm{b}}$ The concentration of the chemicals on $S$. japonicum adult worms in vitro; ${ }^{\mathrm{c}}$ Starting concentration of $1 \mu \mathrm{M}$ when the chemicals killed $100 \%$ of the worms at the concentration of $10 \mu \mathrm{M}$; ${ }^{\mathrm{d}}$ Data only showed the concentration of $100 \mu \mathrm{M}$ when the chemicals have no effect on the worms at 10, 25 and $50 \mu \mathrm{M}$; ${ }^{\mathrm{e}}$ Reported in References [20,21,29] and references therein.

\section{Experimental}

\subsection{Chemistry}

All chemicals were reagent grade and used as purchased. All reactions were performed under an inert atmosphere of dry argon or nitrogen using distilled dry solvent. ${ }^{1} \mathrm{H}-\mathrm{NMR}$ spectra $(400 \mathrm{MHz})$ were recorded on a Bruker AVIII $400 \mathrm{MHz}$ spectrometer. The chemical shifts were reported in (ppm) using 
the 7.26 signal of $\mathrm{CDCl}_{3}\left({ }^{1} \mathrm{H}-\mathrm{NMR}\right)$ as internal standards. Mass spectra (MS) were obtained on a Waters Micromass Platform LCZ Mass Spectrometer.

\subsubsection{Procedure for the Preparation of Compound 1}

A mixture of PZQ (10 g, $32 \mathrm{mmol})$ and $2 \mathrm{~N} \mathrm{HCl}(50 \mathrm{~mL})$ was refluxed overnight. After PZQ was consumed, the reaction solution was cooled to room temperature, neutralized with $\mathrm{NaHCO}_{3}$ (aq.) and then extracted with $\mathrm{DCM} / \mathrm{MeOH}(\mathrm{V} / \mathrm{V} 10 / 1,100 \mathrm{~mL} \times 3)$. The organic layer was washed with water and brine, dried with anhydrous $\mathrm{Na}_{2} \mathrm{SO}_{4}$ and concentrated to get the crude product. The crude product was washed with petroleum ether/EtOAc (V/V 10/1) to yield compound $\mathbf{1}$ (4 g, 62\%) as a yellow solid. ${ }^{1} \mathrm{H}-\mathrm{NMR}\left(\mathrm{CDCl}_{3}\right) \delta:$ 2.73-3.03 (m, 4H), 3.48-3.77 (m, 3H), 4.79-4.89 (m, 2H), 7.13-7.27 (m, 4H); MS (ESI): $m / z$ calcd for $\mathrm{C}_{12} \mathrm{H}_{15} \mathrm{~N}_{2} \mathrm{O}[\mathrm{M}+\mathrm{H}]^{+}: 203.1$, found: 203.4 .

\subsubsection{Procedure for the Preparation of Compound 8}

A stirred solution of compound $1(500 \mathrm{mg}, 2.5 \mathrm{mmol})$ in DCM $(20 \mathrm{~mL})$ was added to trifluoroacetic anhydride $(275 \mu \mathrm{L}, 2 \mathrm{mmol})$ and triethylamine $(275 \mu \mathrm{L}, 2 \mathrm{mmol})$. After the addition, the mixture was stirred at room temperature overnight. The reaction residue was poured into water, and extracted with EtOAc $(50 \mathrm{~mL} \times 3)$. The organic phases were then processed in the usual way and chromatographed (1:1 petroleum ether/EtOAc) to afford compound $8(450 \mathrm{mg}, 62 \%)$ as a white solid. ${ }^{1} \mathrm{H}-\mathrm{NMR}$ $\left(\mathrm{CDCl}_{3}\right) \delta: 2.96(\mathrm{~m}), 3.38(\mathrm{dd}, J=10.8 \mathrm{~Hz}, 14.0 \mathrm{~Hz})($ total $4 \mathrm{H}), 4.00(\mathrm{~d}, J=18.4 \mathrm{~Hz}), 4.26(\mathrm{~d}, J=17.6 \mathrm{~Hz})$ (total 1H), $4.61(\mathrm{dd}, J=14.0 \mathrm{~Hz}, 18.0 \mathrm{~Hz}, 1 \mathrm{H}), 4.84-5.10(\mathrm{~m}, 3 \mathrm{H}), 7.16-7.34(\mathrm{~m}, 4 \mathrm{H}) ; \mathrm{MS}(\mathrm{ESI}): \mathrm{m} / \mathrm{z}$ calcd. for $[\mathrm{M}+\mathrm{H}]^{+}:$299.1, found: 299.2 .

\subsubsection{General Procedure for the Preparation of Derivatives 2-7 and 9-12}

A stirred solution of compound $1(500 \mathrm{mg}, 2.5 \mathrm{mmol})$ in DCM (50 mL) was added to cyclopropanecarbonyl chloride $(420 \mu \mathrm{L}, 3.7 \mathrm{mmol})$ at $0{ }^{\circ} \mathrm{C}$ slowly. After the addition, the mixture was stirred at room temperature overnight. The reaction residue was poured into $\mathrm{NaHCO}_{3}$ (aq.), extracted with DCM $(250 \mathrm{~mL})$, washed with water and brine/dried over $\mathrm{Na}_{2} \mathrm{SO}_{4}$. The organic phases were then processed in the usual way and chromatographed (1:1 petroleum ether/EtOAc) to afforded compound 2 (350 mg, 49\%). ${ }^{1} \mathrm{H}-\mathrm{NMR}\left(\mathrm{CDCl}_{3}\right) \delta: 0.89(\mathrm{~m}, 2 \mathrm{H}), 1.04-1.15(\mathrm{~m}, 2 \mathrm{H}), 1.73(\mathrm{~m}, 1 \mathrm{H}), 2.79-3.02$ $(\mathrm{m}, 4 \mathrm{H}), 4.23(\mathrm{~d}, J=17.2 \mathrm{~Hz}, 1 \mathrm{H}), 4.71(\mathrm{~d}, J=17.6 \mathrm{~Hz}, 1 \mathrm{H}), 4.84-4.87$ (m, 2H), 5.15 (d, $J=12.8 \mathrm{~Hz}$, 1H), 7.20-7.28 (m, 4H); MS (ESI): $\mathrm{m} / \mathrm{z}$ calcd. for $[\mathrm{M}+\mathrm{H}]^{+}:$271.1, found: 271.2. The following compounds were similarly prepared.

2-(Cyclobutanecarbonyl)-2,3,6,7-tetrahydro-1H-pyrazino[2,1-a]isoquinolin-4(11bH)-one (3). Yield = 40\%; ${ }^{1} \mathrm{H}-\mathrm{NMR}\left(\mathrm{CDCl}_{3}\right) \delta: 1.88-2.11(\mathrm{~m}, 2 \mathrm{H}), 2.19-2.27(\mathrm{~m}, 2 \mathrm{H}), 2.32-2.50(\mathrm{~m}, 2 \mathrm{H}), 2.78-3.03(\mathrm{~m}, 4 \mathrm{H})$, $3.15-3.42(\mathrm{~m}, 1 \mathrm{H}), 3.85(\mathrm{~d}, J=18.8 \mathrm{~Hz}), 3.99(\mathrm{~d}, J=17.2 \mathrm{~Hz})($ total $1 \mathrm{H}), 4.27(\mathrm{~d}, J=17.6 \mathrm{~Hz}, 1 \mathrm{H})$, 4.74-4.91 (m, 2H), $5.14(\mathrm{~m}, 1 \mathrm{H}), 7.17-7.33(\mathrm{~m}, 4 \mathrm{H})$; MS (ESI): $\mathrm{m} / \mathrm{z}$ calcd. for $[\mathrm{M}+\mathrm{H}]^{+}: 285.2$, found: 285.3 .

2-(Cyclopentanecarbonyl)-2,3,6,7-tetrahydro-1H-pyrazino[2,1-a]isoquinolin-4(11bH)-one (4). Yield = 43\%; ${ }^{1} \mathrm{H}-\mathrm{NMR}\left(\mathrm{CDCl}_{3}\right) \delta: 1.62-1.96(\mathrm{~m}, 8 \mathrm{H}), 2.78-3.02(\mathrm{~m}, 5 \mathrm{H}), 3.54-3.90(\mathrm{~m}, 1 \mathrm{H}), 4.10(\mathrm{~d}, J=17.2 \mathrm{~Hz})$, 
$4.51(\mathrm{~d}, J=17.2 \mathrm{~Hz})($ total $1 \mathrm{H}), 4.82-4.4 .91(\mathrm{~m}), 5.18(\mathrm{dd}, J=2.8 \mathrm{~Hz}, 13.2 \mathrm{~Hz})($ total $3 \mathrm{H}), 7.16-7.30$ (m, 4H); MS (ESI): $m / z$ calcd. for $[\mathrm{M}+\mathrm{H}]^{+}: 299.2$, found: $299.4[\mathrm{M}+\mathrm{H}]^{+}$.

2-Acetyl-2,3,6,7-tetrahydro-1H-pyrazino[2,1-a]isoquinolin-4(11bH)-one (5). Yield = 43\%; ${ }^{1} \mathrm{H}-\mathrm{NMR}$ $\left(\mathrm{CDCl}_{3}\right) \delta: 2.19(\mathrm{~s}), 2.25$ (s) (total 3H), 2.79-3.01 (m), 3.29-3.35 (m) (total 4H), 3.92 (d, J=18.4 Hz), $4.11(\mathrm{~d}, J=17.6 \mathrm{~Hz})($ total $1 \mathrm{H}), 4.31-4.47(\mathrm{~m}), 4.38(\mathrm{~d}, J=17.6 \mathrm{~Hz})($ total $1 \mathrm{H}), 4.77-4.89(\mathrm{~m}, 2 \mathrm{H})$, 5.13-5.18 (m, 1H), 7.20-7.33 (m, 4H); MS (ESI): $m / z$ calcd. for $[\mathrm{M}+\mathrm{H}]^{+}: 245.1$, found: 245.1 .

2-Isobutyryl-2,3,6,7-tetrahydro-1H-pyrazino[2,1-a]isoquinolin-4(11bH)-one (6). Yield $=43 \%$; ${ }^{1} \mathrm{H}$ NMR $\left(\mathrm{CDCl}_{3}\right) \delta: 1.10(\mathrm{~d}, J=6.8 \mathrm{~Hz}), 1.13(\mathrm{~d}, J=6.8 \mathrm{~Hz})($ total $6 \mathrm{H}), 2.71-2.97(\mathrm{~m}), 3.21(\mathrm{~m})$ (total $5 \mathrm{H}), 3.81(\mathrm{~d}, J=18.4 \mathrm{~Hz}), 4.01-4.09(\mathrm{~m})($ total $1 \mathrm{H}), 4.41(\mathrm{~d}, J=17.2 \mathrm{~Hz}, 1 \mathrm{H}), 4.76(\mathrm{~m}, 2 \mathrm{H}), 5.11$ (dd, $J=2.4 \mathrm{~Hz}, 12.8 \mathrm{~Hz}, 1 \mathrm{H}), 7.12-7.23(\mathrm{~m}, 4 \mathrm{H})$; MS (ESI): $\mathrm{m} / z$ calcd. for $[\mathrm{M}+\mathrm{H}]^{+}:$273.2, found: 273.0.

2-(2-Chloroacetyl)-2,3,6,7-tetrahydro-1H-pyrazino[2,1-a]isoquinolin-4(11bH)-one (7). Yield = 44\%; ${ }^{1} \mathrm{H}-\mathrm{NMR}\left(\mathrm{CDCl}_{3}\right) \delta$ : 2.78-2.96 (m), 3.28-3.34 (m) (total 4H), $3.89(\mathrm{~d}, J=18.4 \mathrm{~Hz}), 4.19-4.26(\mathrm{~m})$ (total $1 \mathrm{H}), 4.19(\mathrm{~s}), 4.15(\mathrm{~s})($ total $2 \mathrm{H}), 4.42-4.46(\mathrm{~m}, 1 \mathrm{H}), 4.77-4.88(\mathrm{~m}, 2 \mathrm{H}), 5.02-5.08(\mathrm{~m}, 1 \mathrm{H}), 7.19-7.28$ $(\mathrm{m}, 4 \mathrm{H}) ; \mathrm{MS}(\mathrm{ESI}): \mathrm{m} / \mathrm{z}$ calcd. for $[\mathrm{M}+\mathrm{H}]^{+}: 279.1 / 281.1$, found: $279.2 / 281.3$.

2-Benzoyl-2,3,6,7-tetrahydro-1H-pyrazino[2,1-a]isoquinolin-4(11bH)-one (9). Yield = 34\%; ${ }^{1} \mathrm{H}-\mathrm{NMR}$ $\left(\mathrm{CDCl}_{3}\right) \delta:$ 2.77-3.10 (m, 4H), 4.06-4.11 (m, 1H), 4.25-4.39 (m, 1H), 4.82-4.99 (m, 2H), 5.25 $(\mathrm{m}, 1 \mathrm{H}), 7.19-7.49(\mathrm{~m}, 9 \mathrm{H})$; MS (ESI): $\mathrm{m} / \mathrm{z}$ calcd for $[\mathrm{M}+\mathrm{H}]^{+}: 307.1$, found: 307.2.

2-Isonicotinoyl-2,3,6,7-tetrahydro-1H-pyrazino[2,1-a]isoquinolin-4(11bH)-one (10). Yield = 29\%; ${ }^{1} \mathrm{H}-\mathrm{NMR}\left(\mathrm{CDCl}_{3}\right) \delta:$ 2.80-3.30 (m, 4H), 4.10-4.27 (m, 2H), 4.81-5.24 (m, 3H), $6.75(\mathrm{~m}), 7.23-7.39$ (m) (total $6 \mathrm{H}), 8.79(2 \mathrm{H})$; MS (ESI): $\mathrm{m} / z$ calcd. for $[\mathrm{M}+\mathrm{H}]^{+}: 308.1$, found: 308.4 .

2-(Thiophene-2-carbonyl)-2,3,6,7-tetrahydro-1H-pyrazino[2,1-a]isoquinolin-4(11bH)-one (11). Yield = 29\%; ${ }^{1} \mathrm{H}-\mathrm{NMR}\left(\mathrm{CDCl}_{3}\right) \delta:$ 2.80-2.85 (m, 1H), 2.90-3.04 (m, 2H), 3.12-3.18 (m, 1H), $4.27(\mathrm{~d}, J=17.6 \mathrm{~Hz}$, $1 \mathrm{H}), 4.86(\mathrm{~m}, 2 \mathrm{H}), 5.03(\mathrm{dd}, J=4.0 \mathrm{~Hz}, 10.8 \mathrm{~Hz}, 1 \mathrm{H}), 5.11(\mathrm{~d}, J=11.2 \mathrm{~Hz}, 1 \mathrm{H}), 7.14(\mathrm{dd}, J=3.6 \mathrm{~Hz}$, $4.8 \mathrm{~Hz}, 1 \mathrm{H}), 7.22-7.30(\mathrm{~m}, 4 \mathrm{H}), 7.46(\mathrm{~d}, J=3.6 \mathrm{~Hz}, 1 \mathrm{H}), 7.57$ (d, $J=4.4 \mathrm{~Hz}, 1 \mathrm{H})$; MS (ESI): $m / z$ calcd. for $[\mathrm{M}+\mathrm{H}]^{+}: 313.1$, found: 313.2 .

2-(Furan-2-carbonyl)-2,3,6,7-tetrahydro-1H-pyrazino[2,1-a]isoquinolin-4(11bH)-one (12). Yield = 50\%; ${ }^{1} \mathrm{H}-\mathrm{NMR}\left(\mathrm{CDCl}_{3}\right) \delta: 2.80-3.19(\mathrm{~m}, 4 \mathrm{H}), 4.29(\mathrm{~m}, 1 \mathrm{H}), 4.87(\mathrm{~d}, J=11.2 \mathrm{~Hz}, 1 \mathrm{H}), 5.01(\mathrm{~d}, J=15.2 \mathrm{~Hz}$, 2H), $5.19(\mathrm{~d}, J=11.2 \mathrm{~Hz}, 1 \mathrm{H}), 6.56(\mathrm{~d}, J=3.2 \mathrm{~Hz}, 1 \mathrm{H}), 7.19(\mathrm{~d}, J=3.6 \mathrm{~Hz}, 1 \mathrm{H}), 7.22-7.31(\mathrm{~m}, 4 \mathrm{H})$, 7.58 (s, 1H); MS (ESI): $m / z$ calcd. for $[\mathrm{M}+\mathrm{H}]^{+}: 297.1$, found: 297.2.

\subsubsection{General Procedure for the Preparation of Derivatives 13-15}

To a solution of compound 1 (305 $\mathrm{mg}, 1.5 \mathrm{mmol})$ in methanol $(10 \mathrm{~mL})$, cyclohexanecarbaldehyde $(186 \mu \mathrm{L}, 1.5 \mathrm{mmol})$ was added at $0{ }^{\circ} \mathrm{C}$, followed by acetic acid $(170 \mu \mathrm{L}, 3.0 \mathrm{mmol})$ addition. The mixture was thus maintained at $0{ }^{\circ} \mathrm{C}$ for $1 \mathrm{~h}$. Then it was heated at $60{ }^{\circ} \mathrm{C}$ for $2 \mathrm{~h}$. After cooled to $0{ }^{\circ} \mathrm{C}$, $\mathrm{NaBH}_{4}(0.45 \mathrm{~g}, 12.0 \mathrm{mmol})$ was added by portions. The reaction mixture was stirred at $60-70{ }^{\circ} \mathrm{C}$ for $12 \mathrm{~h}$, followed by evaporation to remove methanol. The residue was diluted with water $(30 \mathrm{~mL})$ and 
extracted with ethyl acetate $(30 \mathrm{~mL} \times 3)$. The organic phases were then processed in the usual way and chromatographed (2:1 petroleum ether/EtOAc) to afforded compound $13(140 \mathrm{mg}, 32 \%)$ as a white solid. ${ }^{1} \mathrm{H}-\mathrm{NMR}\left(\mathrm{CDCl}_{3}\right) \delta$ : 0.89-0.95 (m, 4H), 1.59-1.83 (m, 6H), 2.23-2.35 (m, 3H), 2.75-2.99 (m, 4H), 3.46-3.60 (m, 3H), 4.80-4.90 (m, 2H), 7.16-7.28 (m, 4H); MS (ESI): m/z calcd. for [M+H] ${ }^{+}$: 299.2, found: 299.3 . The following compounds were similarly prepared.

2-Benzyl-2,3,6,7-tetrahydro-1H-pyrazino[2,1-a]isoquinolin-4(11bH)-one (14). Yield = 35\%; ${ }^{1} \mathrm{H}-\mathrm{NMR}$ $\left(\mathrm{CDCl}_{3}\right) \delta: 2.37(\mathrm{t}, J=10.8 \mathrm{~Hz}, 1 \mathrm{H}), 2.75(\mathrm{~d}, J=14.8 \mathrm{~Hz}, 1 \mathrm{H}), 2.86-2.97(\mathrm{~m}, 3 \mathrm{H}), 3.49-3.59(\mathrm{~m}, 2 \mathrm{H})$, $3.64(\mathrm{AB}, J=13.2 \mathrm{~Hz}, 2 \mathrm{H}), 4.78(\mathrm{~m}, 1 \mathrm{H}), 4.87(\mathrm{~m}, 1 \mathrm{H}), 6.97-7.36(\mathrm{~m}, 9 \mathrm{H})$; MS (ESI): $\mathrm{m} / z$ calcd. for $[\mathrm{M}+\mathrm{H}]^{+}:$293.2, found: 293.4 .

2-(Thiophen-2-ylmethyl)-2,3,6,7-tetrahydro-1H-pyrazino[2,1-a]isoquinolin-4(11bH)-one (15). Yield = 27\%; ${ }^{1} \mathrm{H}-\mathrm{NMR}\left(\mathrm{CDCl}_{3}\right) \delta: 2.37(\mathrm{t}, J=10.4 \mathrm{~Hz}, 1 \mathrm{H}), 2.75(\mathrm{~d}, J=14.8 \mathrm{~Hz}, 1 \mathrm{H}), 2.89-3.02(\mathrm{~m}, 3 \mathrm{H})$, 3.49-3.63 (m, 2H), $3.86(\mathrm{~s}, 2 \mathrm{H}), 4.79(\mathrm{~m}, 1 \mathrm{H}), 4.89(\mathrm{~m}, 1 \mathrm{H}), 6.97-7.29(\mathrm{~m}, 7 \mathrm{H})$. MS (ESI): $\mathrm{m} / z$ calcd. for $[\mathrm{M}+\mathrm{H}]^{+}: 299.1$, found: 299.0 .

\subsubsection{Procedure for the Preparation of Compound 16}

Compound 1 (3.03 g, $15 \mathrm{mmol}$ ) was added to a solution of lithium aluminum hydride (1.14 g, $30 \mathrm{mmol})$ in tetrahydrofuran $(63 \mathrm{~mL})$ and the mixture was refluxed for $10 \mathrm{~h}$ After cooled to $0{ }^{\circ} \mathrm{C}$, the reaction was quenched with aqueous sodium hydroxide $(15 \%, 1.2 \mathrm{~mL})$, and the precipitate was filtrated. The organic phases were then processed in the usual way and chromatographed $\left(10: 1 \mathrm{CH}_{2} \mathrm{Cl}_{2} / \mathrm{MeOH}\right)$ to afforded compound $\mathbf{1 6}(1 \mathrm{~g}, 35 \%)$ as red oil. ${ }^{1} \mathrm{H}-\mathrm{NMR}\left(\mathrm{CDCl}_{3}\right) \delta: 2.39-2.56$ (m, 2H), 2.62-2.68 (m, 2H), 2.83-2.91 (m, 2H), $2.97(\mathrm{dd}, J=2.8 \mathrm{~Hz}, 8.4 \mathrm{~Hz}, 2 \mathrm{H}), 3.07-3.15(\mathrm{~m}, 1 \mathrm{H})$, 3.25 (d, $J=10.4 \mathrm{~Hz}, 1 \mathrm{H}), 3.52$ (dd, $J=2.8 \mathrm{~Hz}, 12.0 \mathrm{~Hz}, 1 \mathrm{H}), 4.58$ (brs, 1H), 7.04-7.13 (m, 4H); MS (ESI): $m / z$ calcd. for $[\mathrm{M}+\mathrm{H}]^{+}: 189.1$, found:189.3.

\subsubsection{General Procedure for the Preparation of Derivatives 17-22}

To a stirred solution of compound $16(500 \mathrm{mg}, 2.7 \mathrm{mmol})$ in DCM (50 mL), cyclohexanecarbonyl chloride $(532 \mu \mathrm{L}, 4.0 \mathrm{mmol})$ was added at $0{ }^{\circ} \mathrm{C}$. The mixture was stirred at room temperature overnight. The reaction was quenched with $\mathrm{NaHCO}_{3}$ (aq.), extracted with DCM $(50 \mathrm{~mL} \times 3)$. The organic phases were then processed in the usual way and chromatographed (1:1 petroleum ether/ EtOAc) to afforded compound $19(350 \mathrm{mg}, 43 \%)$ as white solid. ${ }^{1} \mathrm{H}-\mathrm{NMR}\left(\mathrm{CDCl}_{3}\right) \delta: 1.24-1.87(10 \mathrm{H})$, $2.50(\mathrm{~m}, 1 \mathrm{H}), 2.65(\mathrm{~m}, 2 \mathrm{H}), 2.78(\mathrm{~m}, 1 \mathrm{H}), 2.90-3.13(\mathrm{~m}, 3 \mathrm{H}), 3.28(\mathrm{~m}, 2 \mathrm{H}), 3.44(\mathrm{~m}, 1 \mathrm{H}), 3.92(\mathrm{~d}$, $J=12.4 \mathrm{~Hz}), 4.37(\mathrm{~d}, J=12.8 \mathrm{~Hz})($ total $1 \mathrm{H}), 4.65(\mathrm{~d}, J=13.2 \mathrm{~Hz}), 5.19(\mathrm{~d}, J=12.4 \mathrm{~Hz})($ total $1 \mathrm{H})$, 7.13-7.33 (4H); MS (ESI): $m / z$ calcd. for $[\mathrm{M}+\mathrm{H}]^{+}: 299.2$, found: 299.1. The following compounds were similarly prepared.

Cyclobutyl(3,4,6,7-tetrahydro-1H-pyrazino[2,1-a]isoquinolin-2(11bH)-yl)methanone (17). Yield = 35\%; ${ }^{1} \mathrm{H}-\mathrm{NMR}\left(\mathrm{CDCl}_{3}\right) \delta$ : 1.92-2.01 (m, 2H), 2.15-2.25 (m, 2H), 2.33-2.50 (m, 3H), 2.54-2.64 (m, 2H), 2.75 (m, 1H), 2.87-3.04 (m, 3H), 3.15-3.46 (m, 3H), $3.68(\mathrm{~d}, J=12.8 \mathrm{~Hz}), 4.11-4.18(\mathrm{~m})$ (total 1H), 4.60 $(\mathrm{d}, J=12.0 \mathrm{~Hz}), 5.16(\mathrm{~d}, J=12.4 \mathrm{~Hz})($ total $1 \mathrm{H}), 7.14-7.33(\mathrm{~m}, 4 \mathrm{H})$; MS $(\mathrm{ESI}): \mathrm{m} / \mathrm{z}$ calcd. for $[\mathrm{M}+\mathrm{H}]^{+}$: 271.2, found: 271.3 . 
Cyclopentyl(3,4,6,7-tetrahydro-1H-pyrazino[2,1-a]isoquinolin-2(11bH)-yl)methanone (18). Yield = 32\%; ${ }^{1} \mathrm{H}-\mathrm{NMR}\left(\mathrm{CDCl}_{3}\right) \delta: 1.58-1.98(8 \mathrm{H}), 2.46(\mathrm{~m}, 1 \mathrm{H}), 2.61(\mathrm{~m}, 2 \mathrm{H}), 2.76(\mathrm{~d}, J=17.2 \mathrm{~Hz}, 1 \mathrm{H}), 2.91-3.10$ $(\mathrm{m}, 3 \mathrm{H}), 3.23(\mathrm{~m}, 2 \mathrm{H}), 3.40(\mathrm{~m}, 1 \mathrm{H}), 3.97(\mathrm{~d}, J=12.4 \mathrm{~Hz}), 4.45(\mathrm{~d}, J=12.8 \mathrm{~Hz})($ total $1 \mathrm{H}), 4.66(\mathrm{~d}$, $J=12.8 \mathrm{~Hz}), 5.21(\mathrm{~d}, J=12.8 \mathrm{~Hz})($ total $1 \mathrm{H}), 7.13-7.33(\mathrm{~m}, 4 \mathrm{H})$; MS (ESI): $m / z$ calcd. for $[\mathrm{M}+\mathrm{H}]^{+}$: 285.2, found: 285.1 .

Phenyl(3,4,6,7-tetrahydro-1H-pyrazino[2,1-a]isoquinolin-2(11bH)-yl)methanone (20). Yield = 69\%; ${ }^{1} \mathrm{H}-\mathrm{NMR}\left(\mathrm{CDCl}_{3}\right) \delta:$ 2.40-2.60 (m, 2H), 2.66-2.71 (m, 1H), $2.79(\mathrm{~m}, 1 \mathrm{H}), 2.97(\mathrm{~m}, 2 \mathrm{H}), 3.13-3.33(\mathrm{~m}$, $3 \mathrm{H}), 3.67(\mathrm{~m}), 4.18(\mathrm{~m})($ total 1H), $4.64(\mathrm{~m}), 5.21(\mathrm{~m})($ total 1H), 6.58-7.38 (9H); MS (ESI): $\mathrm{m} / \mathrm{z}$ calcd. for $[\mathrm{M}+\mathrm{H}]^{+}:$293.2, found: 293.2 .

(3,4,6,7-Tetrahydro-1H-pyrazino[2,1-a]isoquinolin-2(11bH)-yl)(thiophen-2-yl)methanone (21). Yield = 44\%; ${ }^{1} \mathrm{H}-\mathrm{NMR}\left(\mathrm{CDCl}_{3}\right) \delta: 2.61(\mathrm{~m}, 2 \mathrm{H}), 2.77(\mathrm{~m}, 1 \mathrm{H}), 3.00-3.08(\mathrm{~m}, 3 \mathrm{H}), 3.18-3.43(\mathrm{~m}, 3 \mathrm{H}), 4.44(\mathrm{brs}, 1 \mathrm{H})$, 5.04 (brs, 1H), 7.12 (t, $J=4.4 \mathrm{~Hz}, 1 \mathrm{H}), 7.15-7.21(\mathrm{~m}, 4 \mathrm{H}), 7.40(\mathrm{~d}, J=2.8 \mathrm{~Hz}, 1 \mathrm{H}), 7.51(\mathrm{~d}, J=5.2$ $\mathrm{Hz}, 1 \mathrm{H})$; MS (ESI): $\mathrm{m} / \mathrm{z}$ calcd. for $[\mathrm{M}+\mathrm{H}]^{+}: 299.1$, found: 299.3 .

2-Chloro-1-(3,4,6,7-tetrahydro-1H-pyrazino[2,1-a]isoquinolin-2(11bH)-yl)ethanone (22). Yield = 36\%; ${ }^{1} \mathrm{H}-\mathrm{NMR}\left(\mathrm{CDCl}_{3}\right) \delta: 2.52-2.78(\mathrm{~m}, 3 \mathrm{H}), 2.95-3.51(\mathrm{~m}, 6 \mathrm{H}), 3.86(\mathrm{~m}), 4.34(\mathrm{~m})$ (total $\left.1 \mathrm{H}\right), 4.15(\mathrm{AB}$, $J=12.0 \mathrm{~Hz}), 4.22(\mathrm{AB}, J=12.0 \mathrm{~Hz})($ total $2 \mathrm{H}), 4.57(\mathrm{~m}),, 5.10(\mathrm{~m})($ total $1 \mathrm{H}), 7.15-7.31(\mathrm{~m}, 4 \mathrm{H})$; MS (ESI): $m / z$ calcd. for $[\mathrm{M}+\mathrm{H}]^{+}: 265.1 / 267.1$, found: $265.2 / 267.1$.

\subsubsection{The Procedure for the Preparation of Compound 24}

A stirred solution of compound $23(1.0 \mathrm{~g}, 3.1 \mathrm{mmol})$ in $\mathrm{HCl}(2 \mathrm{~N}, 10 \mathrm{~mL})$ was heated to $110{ }^{\circ} \mathrm{C}$ overnight. The reaction mixture was neutralized with solid $\mathrm{NaHCO}_{3}$, and extracted with $\mathrm{DCM} / \mathrm{MeOH}$ $(\mathrm{V} / \mathrm{V} 5 / 1,30 \mathrm{~mL} \times 3)$. The organic phases were then processed in the usual way and chromatographed $\left(10: 1 \mathrm{CH}_{2} \mathrm{Cl}_{2} / \mathrm{MeOH}\right)$ to afforded compound $24(500 \mathrm{mg}, 78 \%) .{ }^{1} \mathrm{H}-\mathrm{NMR}\left(\mathrm{CDCl}_{3}\right) \delta: 2.80-2.88(\mathrm{~m}, 3 \mathrm{H})$, 2.95-3.01 (m, 1H), $3.54(\mathrm{~d}, J=17.6 \mathrm{~Hz}, 1 \mathrm{H}), 3.66(\mathrm{~m}, 1 \mathrm{H}), 3.67(\mathrm{~d}, J=17.2 \mathrm{~Hz}, 1 \mathrm{H}), 4.71(\mathrm{~m}, 1 \mathrm{H})$, $5.12(\mathrm{~m}, 1 \mathrm{H}), 6.80(\mathrm{~d}, J=5.2 \mathrm{~Hz}, 1 \mathrm{H}), 7.19(\mathrm{~d}, J=5.2 \mathrm{~Hz}, 1 \mathrm{H})$; MS (ESI): $m / z$ calcd. for $[\mathrm{M}+\mathrm{H}]^{+}$: 209.1, found: 209.2 .

\subsubsection{General Procedure for the Preparation of Compound 25-33}

To a stirred solution of compound $24(208 \mathrm{mg}, 1 \mathrm{mmol})$ in DCM (10 mL), cyclobutanecarbonyl chloride $(136 \mu \mathrm{L}, 1.2 \mathrm{mmol})$ was added and stirred at room temperature overnight. The reaction was quenched with $\mathrm{NaHCO}_{3}$ (aq.), extracted with DCM. The organic phases were then processed in the usual way and chromatographed (1:1 petroleum ether/EtOAc) to afforded compound $\mathbf{2 6}$ (150 mg, 52\%). ${ }^{1} \mathrm{H}-\mathrm{NMR}\left(\mathrm{CDCl}_{3}\right) \delta: 1.92-2.37(\mathrm{~m}, 6 \mathrm{H}), 2.75(\mathrm{t}, J=11.2 \mathrm{~Hz}, 1 \mathrm{H}), 2.84-2.96(\mathrm{~m}, 3 \mathrm{H}), 3.27(\mathrm{~m}, 1 \mathrm{H})$, $3.75(\mathrm{~d}, J=18.8 \mathrm{~Hz}) 3.94(\mathrm{~d}, J=17.6 \mathrm{~Hz})($ total $1 \mathrm{H}), 4.18(\mathrm{~d}, J=12.8 \mathrm{~Hz}), 4.25(\mathrm{~d}$, $J=17.6 \mathrm{~Hz})($ total $1 \mathrm{H}), 4.71(\mathrm{~d}, J=10.4 \mathrm{~Hz}, 1 \mathrm{H}), 4.91(\mathrm{~d}, J=19.2 \mathrm{~Hz}), 5.04(\mathrm{dd}, J=3.2 \mathrm{~Hz}, 12.8 \mathrm{~Hz})$ (total $1 \mathrm{H}), 5.10(\mathrm{dd}, J=2.8 \mathrm{~Hz}, 13.2 \mathrm{~Hz}, 1 \mathrm{H}), 6.81(\mathrm{~d}, J=5.2 \mathrm{~Hz}), 6.91(\mathrm{~d}, J=5.2 \mathrm{~Hz})($ total $1 \mathrm{H}), 7.19$ (d, $J=4.8 \mathrm{~Hz}), 7.23(\mathrm{~d}, J=4.8 \mathrm{~Hz})($ total $1 \mathrm{H})$; MS (ESI): $\mathrm{m} / z$ calcd. for $[\mathrm{M}+\mathrm{H}]^{+}:$291.1, found: 291.2. 
2-(Cyclopentanecarbonyl)-2,3,6,7-tetrahydro-1H-thieno[3',2':3,4]pyrido[1,2-a]pyrazin-4(10bH)-one (25). Yield $=52 \% ;{ }^{1} \mathrm{H}-\mathrm{NMR}\left(\mathrm{CDCl}_{3}\right) \delta: 1.61-1.91(\mathrm{~m}, 8 \mathrm{H}), 2.74(\mathrm{t}, J=11.2 \mathrm{~Hz}, 1 \mathrm{H}), 2.86-2.97(\mathrm{~m}, 4 \mathrm{H})$, $3.76(\mathrm{~d}, J=18.4 \mathrm{~Hz}), 4.04(\mathrm{~d}, J=17.6 \mathrm{~Hz})($ total $1 \mathrm{H}), 4.50(\mathrm{~d}, J=17.6 \mathrm{~Hz}, 1 \mathrm{H}), 4.71(\mathrm{~d}, J=9.2 \mathrm{~Hz}$, $1 \mathrm{H}), 4.93(\mathrm{~d}, J=19.2 \mathrm{~Hz}), 5.04(\mathrm{dd}, J=5.2 \mathrm{~Hz}, 12.8 \mathrm{~Hz})(\operatorname{total} 1 \mathrm{H}), 5.15(\mathrm{dd}, J=2.4 \mathrm{~Hz}, 13.2 \mathrm{~Hz}, 1 \mathrm{H})$, $6.90(\mathrm{~d}, J=4.8 \mathrm{~Hz}, 1 \mathrm{H}), 7.19(\mathrm{~d}, J=5.2 \mathrm{~Hz}, 1 \mathrm{H})$; MS (ESI): $m / z$ calcd. for $[\mathrm{M}+\mathrm{H}]^{+}: 305.1$, found: 305.1 .

2-(Cyclopropanecarbonyl)-2,3,6,7-tetrahydro-1H-thieno[3',2':3,4]pyrido[1,2-a]pyrazin-4(10bH)-one

Yield $=57 \% ;{ }^{1} \mathrm{H}-\mathrm{NMR}\left(\mathrm{CDCl}_{3}\right) \delta: 1.01(\mathrm{~m}, 2 \mathrm{H}), 1.12(\mathrm{~m}, 2 \mathrm{H}), 1.70(\mathrm{~m}, 1 \mathrm{H}), 2.72-2.98(\mathrm{~m}, 4 \mathrm{H}), 4.18(\mathrm{~d}$, $J=17.6 \mathrm{~Hz}, 1 \mathrm{H}), 4.72(\mathrm{~m}, 2 \mathrm{H}), 5.04-5.14(\mathrm{~m}, 2 \mathrm{H}), 6.90$ (d, $J=4.8 \mathrm{~Hz}, 1 \mathrm{H}), 7.19$ (d, $J=4.8 \mathrm{~Hz}, 1 \mathrm{H})$; MS (ESI): $m / z$ calcd. for $[\mathrm{M}+\mathrm{H}]^{+}: 277.1$, found: 276.9 .

2-Isobutyryl-2,3,6,7-tetrahydro-1H-thieno[3',2':3,4]pyrido[1,2-a]pyrazin-4(10bH)-one (28). Yield = 46\%; ${ }^{1} \mathrm{H}-\mathrm{NMR}\left(\mathrm{CDCl}_{3}\right) \delta: 1.15(\mathrm{~d}, J=6.8 \mathrm{~Hz}), 1.17(\mathrm{~d}, J=6.8 \mathrm{~Hz})$, (total $\left.6 \mathrm{H}\right), 2.70-2.80(\mathrm{~m}, 1 \mathrm{H}), 2.86-2.98$ $(\mathrm{m}), 3.16-3.25(\mathrm{~m})(4 \mathrm{H}), 3.77(\mathrm{~d}, J=18.4 \mathrm{~Hz}), 4.06(\mathrm{~d}, J=17.6 \mathrm{~Hz})($ total $1 \mathrm{H}), 4.34-4.40(\mathrm{~m}), 4.48(\mathrm{~d}$, $J=17.6 \mathrm{~Hz})($ total $1 \mathrm{H}), 4.71-4.78(\mathrm{~m}, 1 \mathrm{H}), 4.89-5.06(\mathrm{~m}, 1 \mathrm{H}), 5.15(\mathrm{~d}, J=13.2 \mathrm{~Hz}, 1 \mathrm{H}), 6.90(\mathrm{~d}$, $J=5.2 \mathrm{~Hz}, 1 \mathrm{H}), 7.20(\mathrm{~d}, J=5.2 \mathrm{~Hz}, 1 \mathrm{H})$; MS (ESI): $\mathrm{m} / z$ calcd. for $[\mathrm{M}+\mathrm{H}]^{+}: 279.1$, found: 279.2 .

2-Pivaloyl-2,3,6,7-tetrahydro-1H-thieno[3',2':3,4]pyrido[1,2-a]pyrazin-4(10bH)-one (29). Yield = 76\%; ${ }^{1} \mathrm{H}-\mathrm{NMR}\left(\mathrm{CDCl}_{3}\right) \delta: 1.34(\mathrm{~s}, 9 \mathrm{H}), 2.81-2.97(\mathrm{~m}, 4 \mathrm{H}), 3.96(\mathrm{~d}, J=18.0 \mathrm{~Hz}, 1 \mathrm{H}), 4.76(\mathrm{~m}, 1 \mathrm{H}), 4.82(\mathrm{~d}$, $J=17.6 \mathrm{~Hz}, 1 \mathrm{H}), 5.04(\mathrm{dd}, J=4.8 \mathrm{~Hz}, 12.4 \mathrm{~Hz}, 2 \mathrm{H}), 6.87(\mathrm{~d}, J=5.2 \mathrm{~Hz}, 1 \mathrm{H}), 7.21(\mathrm{~d}, J=5.2 \mathrm{~Hz}, 1 \mathrm{H})$; MS (ESI): $m / z$ calcd. for $[\mathrm{M}+\mathrm{H}]^{+}: 293.1$, found: $293.1[\mathrm{M}+\mathrm{H}]^{+}$.

2-Benzoyl-2,3,6,7-tetrahydro-1H-thieno[3',2':3,4]pyrido[1,2-a]pyrazin-4(10bH)-one (30). Yield = 51\%; ${ }^{1} \mathrm{H}-\mathrm{NMR}\left(\mathrm{CDCl}_{3}\right) \delta:$ 2.86-3.02 (m, 4H), $4.08(\mathrm{~m}, 1 \mathrm{H}), 4.36-4.38(\mathrm{~m}, 1 \mathrm{H}), 4.88(\mathrm{~m}, 1 \mathrm{H}), 5.01(\mathrm{~m}, 1 \mathrm{H})$, 5.19-5.25 (m, 1H), 6.98-7.62 (m, 6H), $8.10(\mathrm{~d}, J=7.6 \mathrm{~Hz}, 1 \mathrm{H})$; MS (ESI): $m / z$ calcd. for $[\mathrm{M}+\mathrm{H}]^{+}$: 313.1, found: 313.2 .

2-(Thiophene-2-carbonyl)-2,3,6,7-tetrahydro-1H-thieno[3',2':3,4]pyrido[1,2-a]pyrazin-4(10bH)-one (31). Yield $=40 \% ;{ }^{1} \mathrm{H}-\mathrm{NMR}\left(\mathrm{CDCl}_{3}\right) \delta: 2.87-2.97(\mathrm{~m}, 4 \mathrm{H}), 4.20-4.24(\mathrm{~m}, 1 \mathrm{H}), 4.85-5.10(\mathrm{~m}, 4 \mathrm{H}), 6.90-7.88$ (m, $5 \mathrm{H})$; MS (ESI): $\mathrm{m} / z$ calcd. for $[\mathrm{M}+\mathrm{H}]^{+}: 319.1$, found: 319.0 .

2-(Furan-2-carbonyl)-2,3,6,7-tetrahydro-1H-thieno[3',2':3,4]pyrido[1,2-a]pyrazin-4(10bH)-one (32). Yield $=46 \% ;{ }^{1} \mathrm{H}-\mathrm{NMR}\left(\mathrm{CDCl}_{3}\right) \delta: 2.85-3.03(\mathrm{~m}, 4 \mathrm{H}), 4.21-4.25(\mathrm{~m}, 1 \mathrm{H}), 4.88(\mathrm{~m}, 1 \mathrm{H}), 5.01-5.07(\mathrm{~m}$, 2H), $5.15(\mathrm{~m}, 1 \mathrm{H}), 6.55(\mathrm{~d}, J=2.8 \mathrm{~Hz}, 1 \mathrm{H}), 6.94$ (brs, 1H), $7.17(\mathrm{~d}, J=3.2 \mathrm{~Hz}, 1 \mathrm{H}), 7.22(\mathrm{~d}, J=5.2 \mathrm{~Hz}$, $1 \mathrm{H}), 7.56(\mathrm{~s}, 1 \mathrm{H})$; MS (ESI): $\mathrm{m} / \mathrm{z}$ calcd. for $[\mathrm{M}+\mathrm{H}]^{+}: 303.1$, found: $303.2[\mathrm{M}+\mathrm{H}]^{+}$.

2-(2-Chloroacetyl)-2,3,6,7-tetrahydro-1H-thieno[3',2':3,4]pyrido[1,2-a]pyrazin-4(10bH)-one (33).

Yield $=76 \% ;{ }^{1} \mathrm{H}-\mathrm{NMR}\left(\mathrm{CDCl}_{3}\right) \delta: 2.82-2.98(\mathrm{~m}), 3.24-3.29(\mathrm{~m})($ total $4 \mathrm{H}), 3.81(\mathrm{~d}, J=18.8 \mathrm{~Hz}), 4.23$ $(\mathrm{d}, J=12.4 \mathrm{~Hz})($ total $1 \mathrm{H}), 4.13(\mathrm{~s}), 4.17$ (s) (total 2H), 4.38-4.46 (m, 1H), 4.75-5.06 (m, 3H), 6.87 (d, $J=5.2 \mathrm{~Hz}), 6.90(\mathrm{~d}, J=5.2 \mathrm{~Hz})($ total $1 \mathrm{H}), 7.22(\mathrm{~d}, J=5.2 \mathrm{~Hz}), 7.24(\mathrm{~d}, J=5.2 \mathrm{~Hz})($ total $1 \mathrm{H})$; MS (ESI): $\mathrm{m} / z$ calcd. for $[\mathrm{M}+\mathrm{H}]^{+}: 285.0 / 287.0$, found: $285.1 / 287.1[\mathrm{M}+\mathrm{H}]^{+}$. 


\subsection{Killing Activity of Compounds 1-33 on S. Japonicum Adult Worms in Vitro [36]}

Stock solutions of compounds 1-33 and praziquantel were prepared by dissolving $1 \mathrm{mg}$ of the drugs in $0.4 \mathrm{ml}$ dimethyl sulfoxide (DMSO) and adding $0.6 \mathrm{~mL}$ RPMI 1640 medium. S. japonicum worms obtained from mice (C57BL/6, female, 22-24 g, each infected with 50 cercariae) were washed in RPMI 1640 medium, kept at pH 7.5 with HEPES $20 \mathrm{mM}$ and supplemented with penicillin (100 UI/mL), streptomycin (100 mg/mL) and 10\% fetal bovine serum (FBS, Gibco) . After washing, 8-15 adult worms were transferred to each well of a 24-well culture plate containing $2 \mathrm{~mL}$ of the same medium. The worms were cultured for 30 to $60 \mathrm{~min}$ at $37{ }^{\circ} \mathrm{C}$ in a humid atmosphere containing $5 \%$ $\mathrm{CO}_{2}$, and then different concentrations of compounds 1-33 $(10,25,50,100 \mu \mathrm{M})$ diluted with RPMI 1640 medium were added. Control worms were treated with equal volumes of RPMI 1640 or DMSO, and worms treated with $10,25,50,100 \mu \mathrm{M}$ praziquantel were also observed. The worm mobility, tegumental alterations and parasite survival were monitored under an inverted microscope (Leica, Wetzlar, Germany) at 24, 48 and $72 \mathrm{~h}$. Parasite death was defined as having no motor activity during 2 min of continuous observation as well as morphological and tegumental alterations. The tests were repeated two times when compouds showed worm killing activity below the concentration of $100 \mu \mathrm{M}$.

\section{Conclusions}

Given the widespread morbidity and mortality derived from schistosomiasis and the possible emergence of PZQ-resistant parasites in the near future, it is undoubtedly urgent to develop new chemotherapy for the disease. In this work, we first reinvestigated the SAR of PZQ analogues at positions 2 and 4, and we identified that the introduction of a chloroacetyl group at position 2 led to compound 7 with higher worm-killing activity against adult S. japonicum than PZQ in vitro. Second, we extended the SAR studies by replacing the aromatic moiety with a thiophene ring, which displayed similar activity as PZQ derivatives, further confirming the respective SAR. Notably, compound $\mathbf{3 3}$ with the chloroacetyl group killed $100 \%$ of the worms at concentrations as low as $10 \mu \mathrm{M}$, which represents a more potent compound than PZQ. The study of these structurally diverse compounds will likely provide meaningful information for the design of new PZQ analogues. After the initial in vitro screening, in vivo activity and toxicity will have to be tested. Design and synthesis of more potent antischistosomal agents are currently undergoing in our laboratory.

\section{Acknowledgments}

This work was supported by the National Natural Science Foundation of China (No. 30972581), the Natural Science Foundation of Jiangsu Province (No. BK2008110), the Health Promotion Project Foundation of Jiangsu Province (No. ZX201108), the National S \& T Major Program (No. 2012ZX 10004-220), and the Fundamental Research Funds for the Central Universities (JUSRP1040). We also thank Sergio C. Chai at St. Jude Children's Research Hospital for his suggestions.

\section{Conflict of Interest}

The authors declare no conflict of interest. 


\section{References}

1. Danso-Appiah, A.; Olliaro, P.L.; Donegan, S.; Sinclair, D.; Utzinger, J. Drugs for treating Schistosoma mansoni infection. Cochrane. Database. Syst. Rev. 2013, 2, CD000528.

2. Gryseels, B.; Polman, K.; Clerinx, J.; Kestens, L. Human schistosomiasis. Lancet 2006, 368, 1106-1118.

3. Zheng, H.; Zhang, L.J.; Zhu, R.; Xu, J.; Li, S.Z.; Guo, J.G.; Xiao, N.; Zhou, X.N. Schistosomiasis situation in People's Republic of China in 2011. Chin. J. Schisto. Control. 2012, 24, 621-626.

4. Caffrey, C.R.; Secor, W.E. Schistosomiasis: From drug deployment to drug development. Curr. Opin. Infect. Dis. 2011, 24, 410-417.

5. Stelma, F.F.; Talla, I.; Sow, S.; Kongs, A.; Niang, M.; Polman, K.; Deelder, A.M.; Gryseels, B. Efficacy and side effects of praziquantel in an epidemic focus of Schistosoma mansoni. Am. J. Trop. Med. Hyg. 1995, 53, 167-170.

6. Wang, W.; Wang, L.; Liang, Y.S. Susceptibility or resistance of praziquantel in human schistosomiasis: a Review. Parasitol. Res. 2012, 111, 1871-1877.

7. Caixeta, S.C.; Magalhaes, L.G.; de Melo, N.I.; Wakabayashi, K.A.; Aguiar Gde, P.; Aguiar Dde, P.; Mantovani, A.L.; Alves, J.M.; Oliveira, P.F.; Tavares, D.C.; et al. Chemical composition and in vitro schistosomicidal activity of the essential oil of Plectranthus neochilus grown in Southeast Brazil. Chem. Biodivers. 2011, 8, 2149-2157.

8. Moraes, J.; Almeida, A.A.; Brito, M.R.; Marques, T.H.; Lima, T.C.; Sousa, D.P.; Nakano, E.; Mendonca, R.Z.; Freitas, R.M. Anthelmintic activity of the natural compound (+)-limonene epoxide against Schistosoma mansoni. Planta Med. 2013, 79, 253-258.

9. Porto, T.S.; da Silva Filho, A.A.; Magalhaes, L.G.; dos Santos, R.A.; Furtado, N.A.; Arakawa, N.S.; Said, S.; de Oliveira, D.C.; Gregorio, L.E.; Rodrigues, V.; et al. Fungal transformation and schistosomicidal effects of pimaradienoic acid. Chem. Biodivers. 2012, 9, 1465-1474.

10. Ndjonka, D.; Rapado, L.N.; Silber, A.M.; Liebau, E.; Wrenger, C. Natural Products as a Source for Treating Neglected Parasitic Diseases. Int. J. Mol. Sci. 2013, 14, 3395-3439.

11. Jao, S.C.; Chen, J.; Yang, K.; Li, W.S. Design of potent inhibitors for Schistosoma japonica glutathione S-transferase. Bioorg. Med. Chem. 2006, 14, 304-318.

12. Rai, G.; Sayed, A.A.; Lea, W.A.; Luecke, H.F.; Chakrapani, H.; Prast-Nielsen, S.; Jadhav, A.; Leister, W.; Shen, M.; Inglese, J.; et al. Structure Mechanism Insights and the Role of Nitric Oxide Donation Guide the Development of Oxadiazole-2-Oxides as Therapeutic Agents against Schistosomiasis. J. Med. Chem. 2009, 52, 6474-6483.

13. Rai, G.; Thomas, C.J.; Leister, W.; Maloney, D.J. Synthesis of oxadiazole-2-oxide analogues as potential antischistosomal agents. Tetrahedron Lett. 2009, 50, 1710-1713.

14. Sayed, A.A.; Simeonov, A.; Thomas, C.J.; Inglese, J.; Austin, C.P.; Williams, D.L. Identification of oxadiazoles as new drug leads for the control of schistosomiasis. J. Nat. Med. 2008, 14, 407-412.

15. Liu, J.; Dyer, D.; Wang, J.; Wang, S.; Du, X.; Xu, B.; Zhang, H.; Wang, X.; Hu, W. 3-Oxoacyl-ACP Reductase from Schistosoma japonicum: Integrated In Silico-In Vitro Strategy for Discovering Antischistosomal Lead Compounds. PLoS One 2013, 8, e64984.

16. Domling, A.; Khoury, K. Praziquantel and schistosomiasis. Chemmedchem 2010, 5, 1420-1434. 
17. Woelfle, M.; Seerden, J.P.; de Gooijer, J.; Pouwer, K.; Olliaro, P.; Todd, M.H. Resolution of praziquantel. PLoS. Negl. Trop. Dis. 2011, 5, e1260.

18. Meyer, T.; Sekljic, H.; Fuchs, S.; Bothe, H.; Schollmeyer, D.; Miculka, C. Taste, a new incentive to switch to (R)-praziquantel in schistosomiasis treatment. PLoS Negl. Trop. Dis. 2009, 3, e357.

19. Cao, H.; Liu, H.; Domling, A. Efficient multicomponent reaction synthesis of the schistosomiasis drug praziquantel. Chemistry 2010, 16, 12296-12298.

20. Liu, H.; William, S.; Herdtweck, E.; Botros, S.; Domling, A. MCR synthesis of praziquantel derivatives. Chem. Biol. Drug. Des. 2012, 79, 470-477.

21. Andrews, P.; Thomas, H.; Pohlke, R.; Seubert, J. Praziquantel. Med. Res. Rev. 1983, 3, 147-200.

22. Patra, M.; Ingram, K.; Pierroz, V.; Ferrari, S.; Spingler, B.; Keiser, J.; Gasser, G. Ferrocenyl derivatives of the anthelmintic praziquantel: Design, synthesis, and biological evaluation. J. Med. Chem. 2012, 55, 8790-8798.

23. Dong, Y.X.; Chollet, J.; Vargas, M.; Mansour, N.R.; Bickle, Q.; Alnouti, Y.; Huang, J.G.; Keiser, J.; Vennerstrom, J.L. Praziquantel analogs with activity against juvenile Schistosoma mansoni. Bioorg. Med. Chem. Lett. 2010, 20, 2481-2484.

24. Patra, M.; Ingram, K.; Pierroz, V.; Ferrari, S.; Spingler, B.; Gasser, R.B.; Keiser, J.; Gasser, G. [(eta(6)-Praziquantel) $\mathrm{Cr}(\mathrm{CO}) 3]$ derivatives with remarkable in vitro anti-schistosomal activity. Chemistry 2013, 19, 2232-2235.

25. Ronketti, F.; Ramana, A.V.; Chao-Ming, X.; Pica-Mattoccia, L.; Cioli, D.; Todd, M.H. Praziquantel derivatives I: Modification of the aromatic ring. Bioorg. Med. Chem. Lett. 2007, 17, 4154-4157.

26. Duan, W.W.; Qiu, S.J.; Zhao, Y.; Sun, H.; Qiao, C.; Xia, C.M. Praziquantel derivatives exhibit activity against both juvenile and adult Schistosoma japonicum. Bioorg. Med. Chem. Lett. 2012, $22,1587-1590$.

27. Wang, Z.X.; Chen, J.L.; Qiao, C. Praziquantel Derivatives with Anti-Schistosomal Activity: Aromatic ring Modification. Chem. Biol. Drug. Des. 2013, 82, 216-225.

28. Sadhu, P.S.; Kumar, S.N.; Chandrasekharam, M.; Pica-Mattoccia, L.; Cioli, D.; Rao, V.J. Synthesis of new praziquantel analogues: Potential candidates for the treatment of schistosomiasis. Bioorg. Med. Chem. Lett. 2012, 22, 1103-1106.

29. Frehel, D.; Maffrand, J.P. Synthesis of the new tricyclic system thieno[3',2':3,4]pyrido[1,2a]pyrazin-4-one. Heterocycles 1984, 22, 143-149.

30. Todd, M.H.; Ndubaku, C.; Bartlett, P.A. Amino acid derived heterocycles: Lewis acid catalyzed and radical cyclizations from peptide acetals. J. Org. Chem. 2002, 67, 3985-3988.

31. Bissinger, E.M.; Heinke, R.; Spannhoff, A.; Eberlin, A.; Metzger, E.; Cura, V.; Hassenboehler, P.; Cavarelli, J.; Schule, R.; Bedford, M.T.; et al. Acyl derivatives of p-aminosulfonamides and dapsone as new inhibitors of the arginine methyltransferase hPRMT1. Bioorg. Med. Chem. 2011, 19, 3717-3731.

32. Kim, D.J.; Reddy, K.; Kim, M.O.; Li, Y.; Nadas, J.; Cho, Y.Y.; Kim, J.E.; Shim, J.H.; Song, N.R.; Carper, A.; et al. (3-Chloroacetyl)-indole, a Novel allosteric AKT inhibitor, Suppresses colon cancer growth in vitro and in vivo. Cancer Prev. Res. 2011, 4, 1842-1851.

33. Nam, K.N.; Koketsu, M.; Lee, E.H. 5-Chloroacetyl-2-amino-1,3-selenazoles attenuate microglial inflammatory responses through NF-kappa B inhibition. Eur. J. Pharmacol. 2008, 589, 53-57. 
34. Dezube, B.J.; Von Roenn, J.H.; Holden-Wiltse, J.; Cheung, T.W.; Remick, S.C.; Cooley, T.P.; Moore, J.; Sommadossi, J.P.; Shriver, S.L.; Suckow, C.W.; et al. Fumagillin analog in the treatment of Kaposi's sarcoma: A phase I AIDS Clinical Trial Group Study. J. Clin. Oncol. 1998, 16, 1444-1449.

35. Kruger, E.A.; Figg, W.D. TNP-470: An Angiogenesis inhibitor in clinical development for cancer. Expert Opin. Invest. Drug 2000, 9, 1383-1396.

36. Song, L.J.; Li, J.H.; Xie, S.Y.; Qian, C.Y.; Wang, J.; Zhang, W.; Yin, X.R.; Hua, Z.C.; Yu, C.X. Thioredoxin Glutathione Reductase as a Novel Drug Target: Evidence from Schistosoma japonicum. PLoS One 2012, 7, e31456.

Sample Availability: Samples of the compounds 1-33 are available from the authors.

(C) 2013 by the authors; licensee MDPI, Basel, Switzerland. This article is an open access article distributed under the terms and conditions of the Creative Commons Attribution license (http://creativecommons.org/licenses/by/3.0/). 